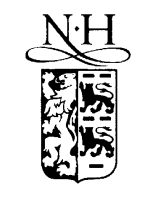

Nuclear Instruments and Methods in Physics Research B 192 (2002) 301-310

www.elsevier.com/locate/nimb

\title{
Interaction of ambient nitrogen gas and laser ablated carbon plume: Formation of $\mathrm{CN}$
}

\author{
R.K. Thareja ${ }^{a, *}$, R.K. Dwivedi ${ }^{\text {b,1 }}$, K. Ebihara ${ }^{\text {a }}$ \\ ${ }^{a}$ Department of Electrical and Computer Engineering, Kumamoto University, Kurokami 2-39-1, Kumamoto 860-8555, Japan \\ b Department of Physics and Centre for Laser Technology, Indian Institute of Technology Kanpur, Kanpur 208016 (UP), India
}

Received 31 October 2000; received in revised form 25 September 2001

\begin{abstract}
Pulsed laser ablated carbon plasma in a nitrogen background gas in the pressure range from 10 mTorr to 100 Torr is investigated by optical emission spectroscopy using 1.064 and $0.355 \mu \mathrm{m}$ laser wavelengths. $\mathrm{C}_{2}$ and $\mathrm{CN}$ emission bands dominated the emitted spectrum at all ambient gas pressures at low incident laser intensity commonly used for pulsed laser deposition of thin films. The light intensity of $\mathrm{C}_{2}$ and $\mathrm{CN}$ band heads is highly dependent on laser wavelength, laser energy, and ambient gas pressures. Emission from $\mathrm{N}_{2}^{+}$was detected at all nitrogen pressures. N I and N II are also observed in the emitted spectrum at the background gas pressure greater than 1 Torr. The vibrational temperature of $\mathrm{CN}$ species is found to be greater than that of $\mathrm{C}_{2}$ species. The mechanism of formation of $\mathrm{C}_{2}$ and $\mathrm{CN}$ molecules is discussed. (C) 2002 Published by Elsevier Science B.V.
\end{abstract}

Keywords: Carbon plasma; Laser-plasma; Spectroscopy; $\mathrm{C}_{2}$ and $\mathrm{CN}$ bands; Thin film

\section{Introduction}

In recent years, pulsed laser deposition (PLD) has become an attractive technique for depositing thin films of a wide variety of materials [1,2]. In spite of the well known established and recognized potentials of the technique, the phenomena involved are not completely understood and the film deposition is partly being done in an empirical

\footnotetext{
${ }^{*}$ Corresponding author. Tel.: +81-96-342-3619; fax: +91512-59-7766.

E-mail addresses: thareja@iitk.ac.in, tharejark@hotmail. com (R.K. Thareja).

${ }^{1}$ Present address: Christ Church College, Kanpur.
}

manner. The growth process may be supplemented by a passive or reactive gas or ion source, which may affect the ablation plume species in the gas phase or the surface reaction. For ablation in reactive gases like oxygen and nitrogen simple oxide and nitride molecules are also formed in the expanding ablation plume resulting in oxide and nitride films on the substrate [3-7]. Among the several characteristics that distinguish PLD from other film growth techniques is congruent deposition, and hence it has been used to grow superconducting superlattices and superconducting device structures [8]. Recently, combinatorial methods, an approach in which a large number of materials are synthesized, processed and screened 
for specific properties have been applied to the discovery and optimization of several functional materials $[9,10]$.

It is now well accepted that laser produced plasmas play an essential role in determining the ultimate characteristics of the deposited films. The characteristics of the pulsed laser induced plasma such as density, temperature and velocity are greatly influenced by various factors such as laser wavelength, laser irradiance, angle of incidence, irradiated spot size, ambient gas pressure and type, and target properties etc. $[1,2,11,12]$. Depending on the laser power and pulse duration the laserablated plume may consist of atoms/ions in the excited states or clusters [13]. Laser ablated plasmas in a background gas are being widely used for nano-particle production [14]. Laser created carbon plasmas in an ambient atmosphere have been used to deposit a variety of thin films such as diamond-like carbon, fullerenes and superhard $\beta$-phase carbon nitride $\left(\beta-\mathrm{C}_{3} \mathrm{~N}_{4}\right)$ etc. $[12,15,16]$. Ablation of aluminum and titanium in presence of nitrogen gas has also been studied to understand the role of plasma parameters in the formation of nitride thin films $[17,18]$. The quality of diamond like films deposited in presence of nitrogen gas is believed to degrade with increase in nitrogen gas pressure. It is argued that both multiple scattering and associative reactions of the ablated species with ambient gas molecules contribute to the film. The quality of the film obtained depends on the distribution of internal energy and kinetic energy of ions, neutrals or clusters in the plasma plume. Hence the knowledge of the behavior of the plume species ejected from laser ablated target surface is a key issue for understanding the laser ablation process and deposition of thin films.

Generally, the diagnostics of PLD plume expansion and its dynamics is carried out using optical emission spectroscopy (OES), absorption spectroscopy, laser induced fluorescence, time of flight mass spectroscopy, fast photography and electrostatic ion probes etc. [6,11,19-21]. Among all these techniques OES is preferred mainly because of its ease of operation and of non-intrusive nature. It provides information about the species emitted and their evolution in an expanding laserinduced plasma plume. Although laser ablation under various conditions has been studied, more work is still needed to understand laser ablation and gas dynamics of the plume. In the present work we have studied spatially and temporally resolved optical emission characteristics of laser ablated carbon plasma in nitrogen atmosphere at low laser energies. The paper is organized as follows. Section 2 gives the experimental set up and parameters of the study. Physical processes necessary to understand laser ablated plasmas and correlation with the deposited film are summarized in Section 3. In Section 4 we discuss the results of our study on interaction of laser ablated carbon species with the background gas. Conclusions are given in Section 5.

\section{Experimental setup}

The experimental setup used in the present work is similar to the one reported elsewhere [13]. The laser ablation of graphite was performed using 1.064 and $0.355 \mu \mathrm{m}$ wavelength of a Nd:YAG (DCR-4G, Laser Spectra) laser with a Gaussian limited mode structure delivering up to $1 \mathrm{~J}$ of energy at its fundamental in $8 \mathrm{~ns}$ (full width at half maximum, FWHM) with $10 \mathrm{~Hz}$ repetition rate. Laser radiation was focused using a spherical lens on to a graphite target (density $1.83 \mathrm{~g} / \mathrm{cm}^{3}$ ) mounted in the vacuum chamber that could be evacuated to a pressure better than $10^{-4}$ Torr. The laser beam was incident normal to the surface and the irradiated spot at the target was of diameter $260 \mu \mathrm{m}$. The target was rotated continuously so as to allow each laser pulse to interact with a new surface area. The vacuum chamber was purged several times before filling the gas into it for performing the experiment. The experiment was carried out at various nitrogen gas pressures ranging from 10 mTorr to 100 Torr. The plasma emission was imaged onto the slit of a monochromator (HRS2, Jobin Yvon) so as to have one to one correspondence with the plasma and its image. The output from the monochromator was detected with a photo-multiplier tube (PMT) (IP28, Hamamatsu) and recorded on a strip chart recorder or displayed on the screen of the storage oscilloscope (TS-8123, 
Iwatsu). The signals were digitized and fed to a personal computer for further data processing.

\section{Physical processes}

PLD is described as a three step process (i) laser-target interaction i.e. absorption of the laser energy by the target leading to a rise in temperature and subsequent vaporization of the target, (ii) material ejection from the target and the formation of plasma plume which expands in vacuum or background gas, (iii) plasma-substrate interaction leading to film deposition. Each of these processes plays a crucial role in defining the quality of the deposited films. In this section we summarize the important general features of PLD. As far as possible the discussion given below is material unspecific.

When laser radiation is absorbed in the surface of material, the electromagnetic energy is immediately converted into electronic excitation in the form of plasmons, unbound electrons, and in the case of insulators, excitons. The excited electrons transfer their energy to the lattice within a few picoseconds and heating begins within the optical absorption depth of the material $1 / \alpha$, where $\alpha$ is the optical absorption coefficient. If the thermal diffusion length, given by $l_{T}=2\left(D \tau_{\mathrm{p}}\right)^{1 / 2}$, where $D$ is the thermal diffusion constant, is smaller than $1 / \alpha$, the bulk will be heated down to $1 / \alpha$, independent of the pulse width. This condition should be satisfied in order to have congruent evaporation of multielemental targets. However, in metals $1 / \alpha \ll l_{T}$, absorption depths are typically $10 \mathrm{~nm}$. Also for ablation using a ns pulse, the nascent vapor cloud may get ionized before the laser pulse is over. The electrons will transfer their energy to the lattice within a few picoseconds resulting in heating of the ablation layer. Therefore, the bulk of material under the plasma is largely screened from the remainder of the laser pulse, which is partly absorbed by the plasma as it becomes increasingly ionized [22]. This process known as laser supported absorption produces plasma species with high kinetic energy ranging between $1 \mathrm{eV}$ and more than $100 \mathrm{eV}$. For materials with small value of absorption depth (a high value of $\alpha$ ) very hot species and lower ablation yields are expected. These highly energetic plasma species provide the possibility of surface processes far from thermal equilibrium. The effective thermal diffusion length is reduced to $l_{T}^{\text {eff }}=2\left(D \tau_{\text {eff }}\right)^{1 / 2}$ where $\tau_{\text {eff }}$ is the time needed to create an erosion plasma after the start of the laser pulse.

Absorption of radiation takes place by the process of inverse bremsstrahlung with an absorption coefficient [23] in $\mathrm{cm}^{-1}$ of

$K_{v}=3.69 \times 10^{8}\left\{\frac{Z^{3} n_{i}^{2}}{T^{1 / 2} v^{3}}\right\}\left[1-\exp \left(-\frac{h v}{k_{\mathrm{B}} T}\right)\right]$

where $n_{i}$ is the density of ions in $\mathrm{cm}^{-3}, T$ is the gas temperature in $\mathrm{K}$ and $v$ is the frequency (in $\mathrm{Hz}$ ) of incident light, $k_{\mathrm{B}}$ is Boltzmann constant, the factor $\left[1-\exp \left(-h v / k_{\mathrm{B}} T\right)\right]$ gives the loss due to stimulated emission. For the Nd:YAG laser of wavelength $1.06 \mu \mathrm{m},\left[1-\exp \left(-h v / k_{\mathrm{B}} T\right)\right]=1$ for $T \ll$ $13,000 \mathrm{~K}$ and close to $h v / k_{\mathrm{B}} T$ for $T \gg 13,000 \mathrm{~K}$. As the vapors cloud absorbs incident radiation it becomes hotter, more ionized and starts absorbing more efficiently. A general feature of ablation plasmas (also known as 'plumes') is their high ion and electron temperatures of the order of several thousand Kelvin, and their high degree of ionization. The high temperature affects the degree of ionization of the plume in accordance with Saha equation [24].

Starting with the initial work of Dawson et al. [25] several studies [26-29] on the effect of a background gas on expanding laser ablated plumes have shown that a plume interacts hydrodynamically with the background gas, undergoes a transition to a stable shock front as the material scatters off the background gas, becomes delayed, and coalesces with the slower moving material. Shock wave, blast wave, and drag force models have been used to explain the dynamics of the expanding plumes. Experimental results are best explained by shock wave and drag force models at large time [30-32]. The front propagates with gradually decreasing velocity towards a substrate on which film growth occurs. Experiments on PLD in the presence of an ambient gas have shown that the deposition rate depends on parameters of the ambient gas. 


\section{Results and discussion}

The optical emission spectra of laser-ablated carbon in the presence of nitrogen gas pressures ranging from $10 \mathrm{mTorr}$ to 100 Torr were recorded at various distances from the target surface. The plasma emission from the target at higher laser intensities was dominated by various atomic/ionic species of C IV to C I. At low laser intensities generally employed for PLD the plasma emission was dominated by $\mathrm{CN}$ violet $\left(\mathrm{B}^{2} \Sigma^{+}-\mathrm{X}^{2} \Sigma^{+}\right)$bands of the sequence $\Delta v=-1,0,1$ and $\mathrm{C}_{2}$ Swan bands (c $\left.{ }^{1} \Pi_{\mathrm{g}}-\mathrm{a}^{3} \Pi_{\mathrm{u}}\right)$ of the sequence $\Delta v=-1$ [33]. The emission from C I, C II, $\mathrm{N}_{2}^{+}$, N I and N II was also observed. No emission was observed when radiation was focused in the chamber without the target.
A typical emission spectrum of the carbon plume in the spectral range $350-400 \mathrm{~nm}$ recorded at a distance of $4 \mathrm{~mm}$ from the target surface at 100 mTorr nitrogen using laser intensity of $1.18 \times 10^{10} \mathrm{~W} \mathrm{~cm}^{-2}$ of $1.064 \mu \mathrm{m}$ radiation is shown in Fig. 1. The emission lines were identified using the information available in the literature [34,35]. The figure shows prominent $\mathrm{CN}$ violet bands $\left(\mathrm{B}^{2} \Sigma^{+}-\mathrm{X}^{2} \Sigma^{+}\right)$of $\Delta v=0$ sequence consisting of band heads (0-0) at $388.3 \mathrm{~nm},(1-1)$ at $387.1 \mathrm{~nm}$, $(2-2)$ at $386.2 \mathrm{~nm},(3-3)$ at $385.5 \mathrm{~nm}$ and (4-4) at $385.1 \mathrm{~nm}$, and for $\Delta v=1$, the bands (1-0) at 359.0 $\mathrm{nm},(2-1)$ at $358.6 \mathrm{~nm}$ and (3-2) at $358.4 \mathrm{~nm}$. In addition, intense emission from $\mathrm{N}_{2}^{+}\left(\mathrm{B}^{2} \Sigma_{\mathrm{u}}^{+}-\mathrm{X}^{2}\right.$ $\left.\Sigma_{\mathrm{g}}^{+}\right), \Delta v=0$ sequence with band head $(0-0)$ at $391.4 \mathrm{~nm}$ and $\Delta v=1$ sequence with band heads (1-0) at $358.2 \mathrm{~nm},(2-1)$ at $356.4 \mathrm{~nm},(3-2)$ at

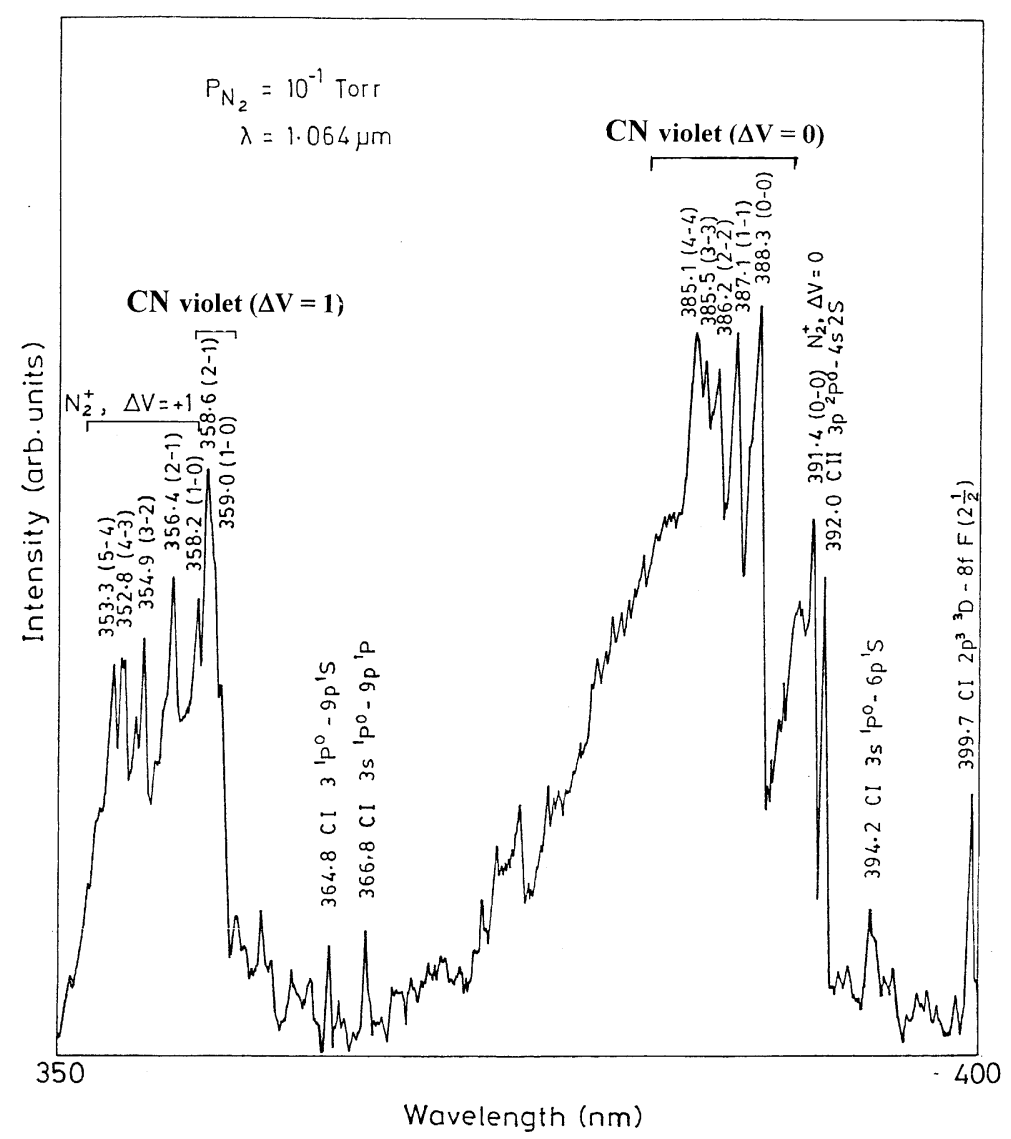

Fig. 1. Emission spectrum of carbon plasma recorded at a distance of $4 \mathrm{~mm}$ from the target surface at $100 \mathrm{mTorr}$ of nitrogen gas pressure at laser intensity of $1.18 \times 10^{10} \mathrm{~W} \mathrm{~cm}^{-2}$ of $1.064 \mu \mathrm{m}$ radiation. 
$354.9 \mathrm{~nm},(4-3)$ at $352.8 \mathrm{~nm}$ and (5-4) at $353.3 \mathrm{~nm}$ are also present. A few C I and C II lines are also seen in the spectrum. Fig. 2 shows the emission spectrum of the plasma in the spectral range 410 $425 \mathrm{~nm}$ at 1 Torr of nitrogen gas pressure. $\mathrm{CN}$ violet bands of $\Delta v=-1$ sequence with band heads $(0-1)$ at $421.6 \mathrm{~nm},(1-2)$ at $419.7 \mathrm{~nm},(2-3)$ at 418.1 $\mathrm{nm},(3-4)$ at $416.8 \mathrm{~nm},(4-5)$ at $415.8 \mathrm{~nm}$ and (5-6) at $415.2 \mathrm{~nm}$ dominate the spectrum. The intensity of all the emitted species increases with increase of background $\mathrm{N}_{2}$ gas pressure. A detailed investigation on $\mathrm{CN}$ bands formation was undertaken to understand their role in PLD. Fig. 3 shows the intensity of (0-0) $\mathrm{CN}$ band head at $388.3 \mathrm{~nm}$ as a function of laser intensity at various nitrogen gas pressures at $6 \mathrm{~mm}$ from the target surface. Every point in the figures to follow is an average of five observations. The band head intensity increases when the $\mathrm{N}_{2}$ gas pressure is increased from 10 mTorr to 1 Torr but decreases with further increase in pressure up to 100 Torr. Similar to earlier reports we did not observe $\mathrm{CN}$ emission at very low pressure [36]. The incorporation of the ambient gas helps to cool the molecular species and increases the recombination rate. The radiative and three body recombination rates have temper-

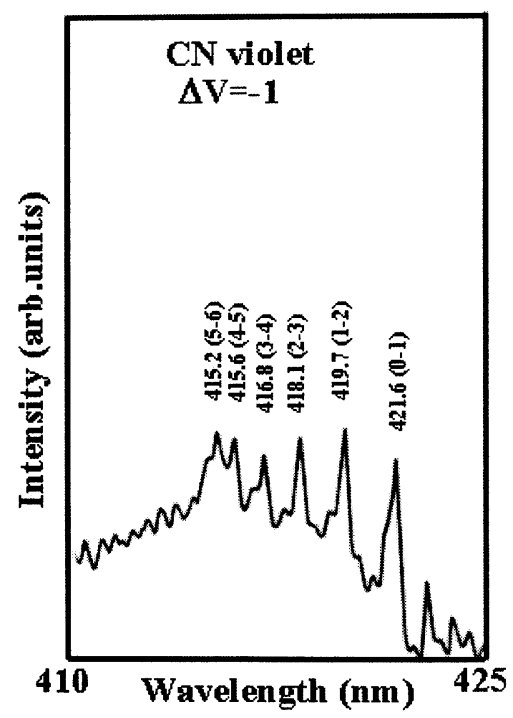

Fig. 2. Emission spectrum ( $\mathrm{CN}$ violet band, $\Delta v=-1)$ of carbon plasma at 1 Torr pressure of nitrogen gas at laser intensity of $1.18 \times 10^{10} \mathrm{~W} \mathrm{~cm}^{-2}$ of $1.064 \mu \mathrm{m}$ radiation.
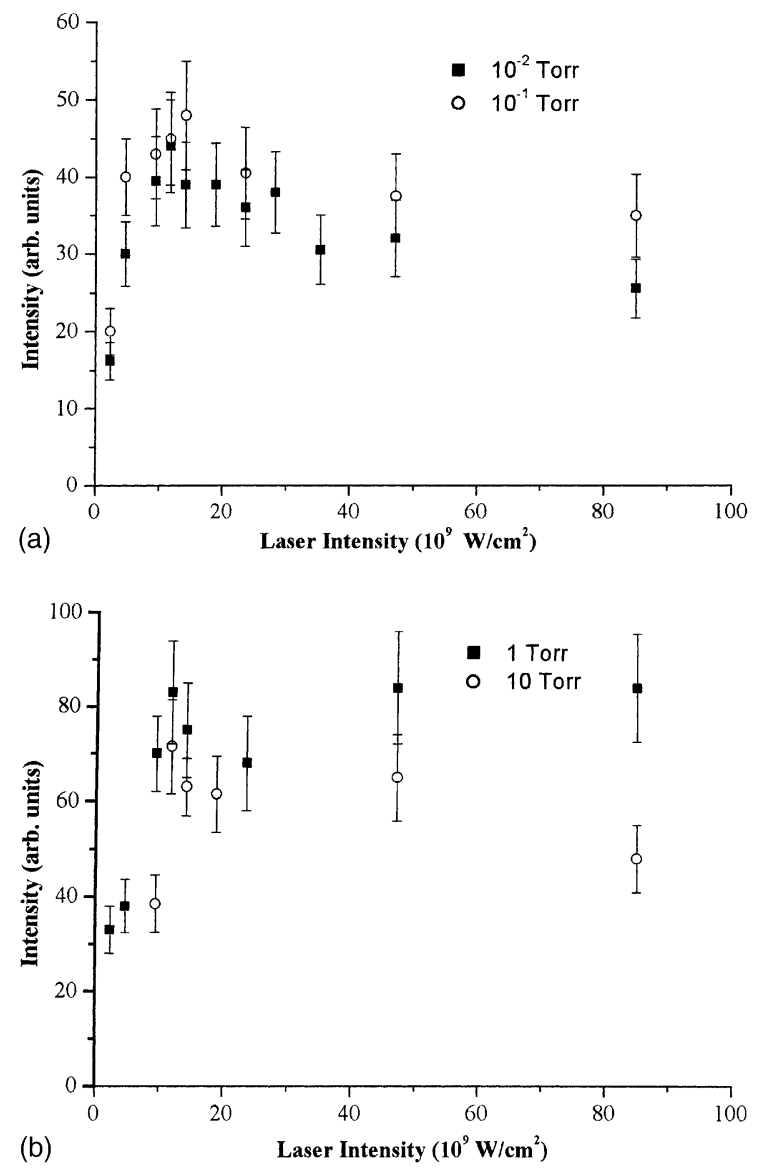

Fig. 3. Variation of intensity of $\mathrm{CN}$ violet band head (0-0) at $388.3 \mathrm{~nm}$ with laser intensity of $1.064 \mu \mathrm{m}$ radiation recorded at a distance of $6 \mathrm{~mm}$ from the target surface and $\mathrm{N}_{2}$ gas pressures of (a) $10^{-2}$ and $10^{-1}$ Torr and (b) 1 and 10 Torr.

ature dependence of $T^{-3 / 4}$ and $T^{-9 / 2}$, respectively [37]. However, at higher pressure the rapid fall observed in intensity may be due to dissociation of nitrogen gas (note the difference in observed spectrum in Figs. 1 and 7) or the decrease in $\mathrm{C}_{2}$ due to cooling of plasma, discussed later. It can also be seen from the figure that the band head intensity first increases with energy, attains a maximum value and then decreases. The dependence of $\mathrm{CN}$ band head intensity as a function of distance from the target surface is shown in Fig. 4. The band head intensity in vacuum decreases as the distance from the target surface is increased. However, in presence of nitrogen ambient the intensity is found to peak at a certain distance along 


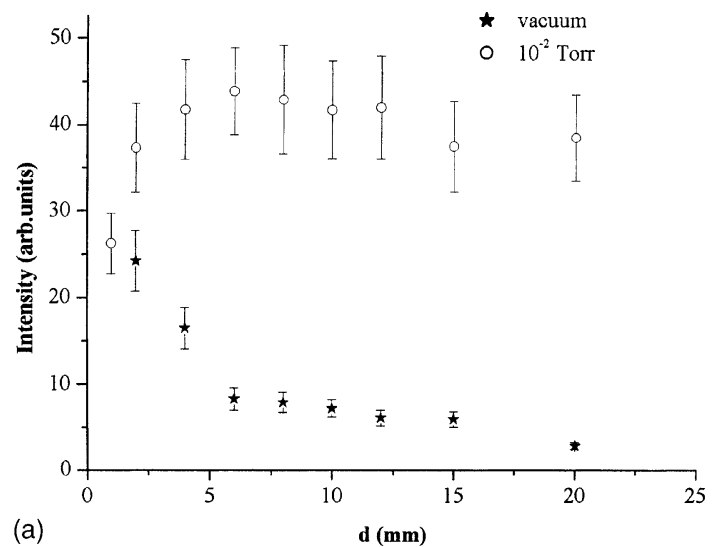

(a)
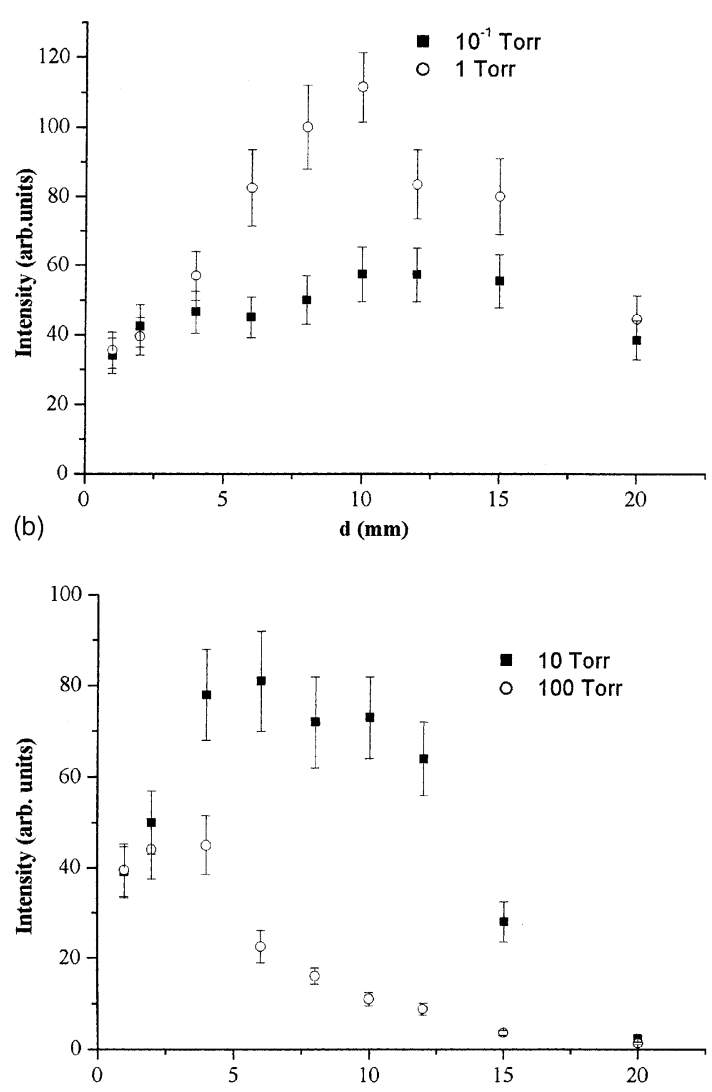

(c)

d (mm)

Fig. 4. Variation of intensity of $\mathrm{CN}$ violet band head $(0-0)$ at $388.3 \mathrm{~nm}$ with distance from the target at laser intensity of $1.18 \times 10^{10} \mathrm{~W} \mathrm{~cm}^{-2}$ of $1.064 \mu \mathrm{m}$ radiation and $\mathrm{N}_{2}$ gas pressure of (a) vacuum ( $\sim 10^{-5}$ Torr) and $10^{-2}$ Torr, (b) $10^{-1}$ and 1 Torr and (c) 10 and 100 Torr. the target surface normal. This depends on density of $\mathrm{C}_{2}$ and pressure of nitrogen gas, discussed later. Due to collisions the $\mathrm{CN}$ band emission profiles also become broader in presence of ambient gas.

We have earlier reported $\mathrm{C}_{2}$ emission from laser ablated carbon using various harmonics of $\mathrm{Nd}$ :YAG laser in presence of helium and argon gases [38]. Fig. 5 shows the behavior of $\mathrm{C}_{2}$ and $\mathrm{CN}$ bands as a function of distance from the target surface at 100 mTorr of nitrogen gas pressure at laser intensity of $1.18 \times 10^{10} \mathrm{~W} \mathrm{~cm}^{-2}$ of $1.064 \mu \mathrm{m}$ radiation. The intensity of $\mathrm{C}_{2}$ bands is found to be much smaller than that of $\mathrm{CN}$ bands. $\mathrm{C}_{2}$ emission has a narrow profile with a maximum close to the target surface and diminishes fast with distance from the target surface. $\mathrm{CN}$ emission has a broad profile with a maximum at a distance larger than that of $\mathrm{C}_{2}$. The different spatial profile of $\mathrm{C}_{2}$ and $\mathrm{CN}$ suggests a different formation mechanism of these species.

In addition to the $\mathrm{C}_{2}$ and $\mathrm{CN}$ bands, $(0-0)$ band of $\mathrm{N}_{2}^{+}$is also observed. The $(0-0)$ band head intensity of $\mathrm{N}_{2}^{+}$at $391.4 \mathrm{~nm}$ as a function of distance at various nitrogen gas pressures is shown in Fig. 6. The intensity of band head shows a maximum at some distance from the target surface and decreases on either side of it. Also, these bands are found to be prominent up to 1 Torr pressure only. The intensity of these bands reduces drastically at pressures beyond 1 Torr of nitrogen gas. The

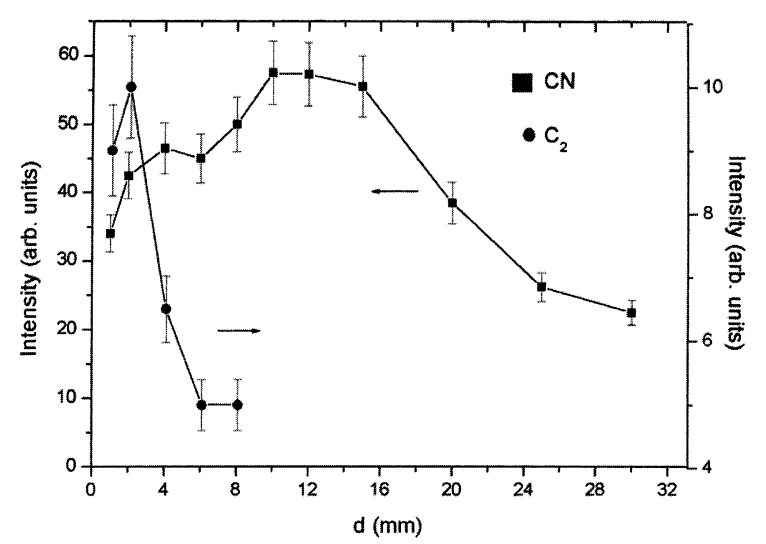

Fig. 5. Behavior of $\mathrm{C}_{2}$ and $\mathrm{CN}$ with distance from the target surface at 100 mTorr of $\mathrm{N}_{2}$ gas pressure at laser intensity of $1.18 \times 10^{10} \mathrm{~W} \mathrm{~cm}^{-2}$ of $1.064 \mu \mathrm{m}$ radiation. 
presence of $\mathrm{N}_{2}^{+}$in the plasma plume can be attributed to the fast electrons impact ionization and excitation of ambient nitrogen gas. The plasma emission showed an altogether different behavior above 1 Torr of pressure. Fig. 7 shows the spec-

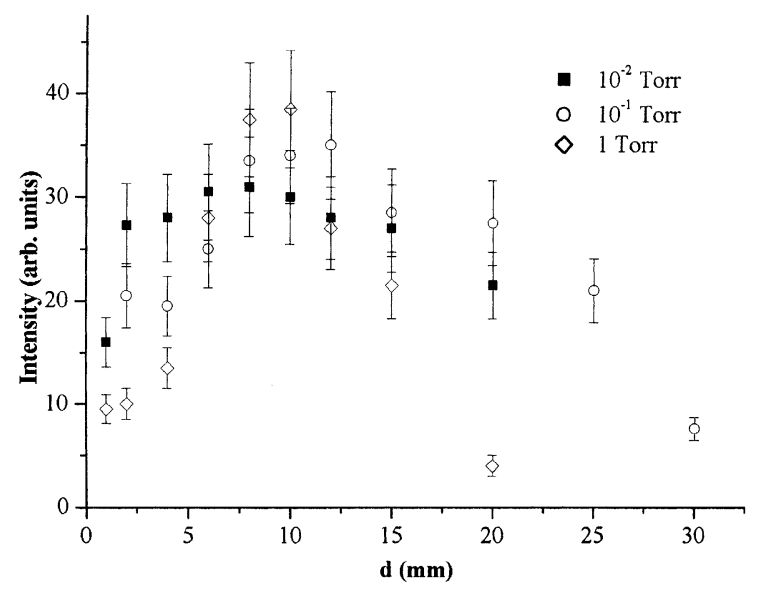

Fig. 6. Dependence of intensity of $\mathrm{N}_{2}^{+}(0-0)$ at $391.4 \mathrm{~nm}$ on distance from the target surface. The laser intensity was $1.18 \times 10^{10} \mathrm{~W} \mathrm{~cm}^{-2}$ of $1.06 \mu \mathrm{m}$ radiation. trum of the plasma plume at 10 Torr $\mathrm{N}_{2}$ gas recorded at a distance of $10 \mathrm{~mm}$ from the target surface. The spectral lines corresponding to N I and $\mathrm{N}$ II are observed in addition to the $\mathrm{CN}$ bands. The spatial evolution of N I and N II was found similar to that of C I and C II. This may be due to the fact that these species are formed by dissociation and ionization of gas molecules by collisions in plasma plume.

To see the effect of incident laser wavelength on $\mathrm{CN}$ bands formation, the emission spectra of the $\mathrm{CN}$ violet band were also recorded using $0.355 \mu \mathrm{m}$ laser wavelength at various nitrogen gas pressures. The observed intensity of the bands was larger at $0.355 \mu \mathrm{m}$ irradiation compared to $1.064 \mu \mathrm{m}$. This probably is due to difference in optical penetration depth that decreases with the wavelength [23] as discussed in Section 3. The smaller volume of interaction results in a more effective coupling of radiation. The vibrational temperature of $\mathrm{CN}$ and $\mathrm{C}_{2}$ molecules was estimated at various ambient gas pressures using different laser energies. Band head intensities of $\mathrm{CN}$ violet and $\mathrm{C}_{2}$ Swan bands were used to estimate the vibrational temperature. The

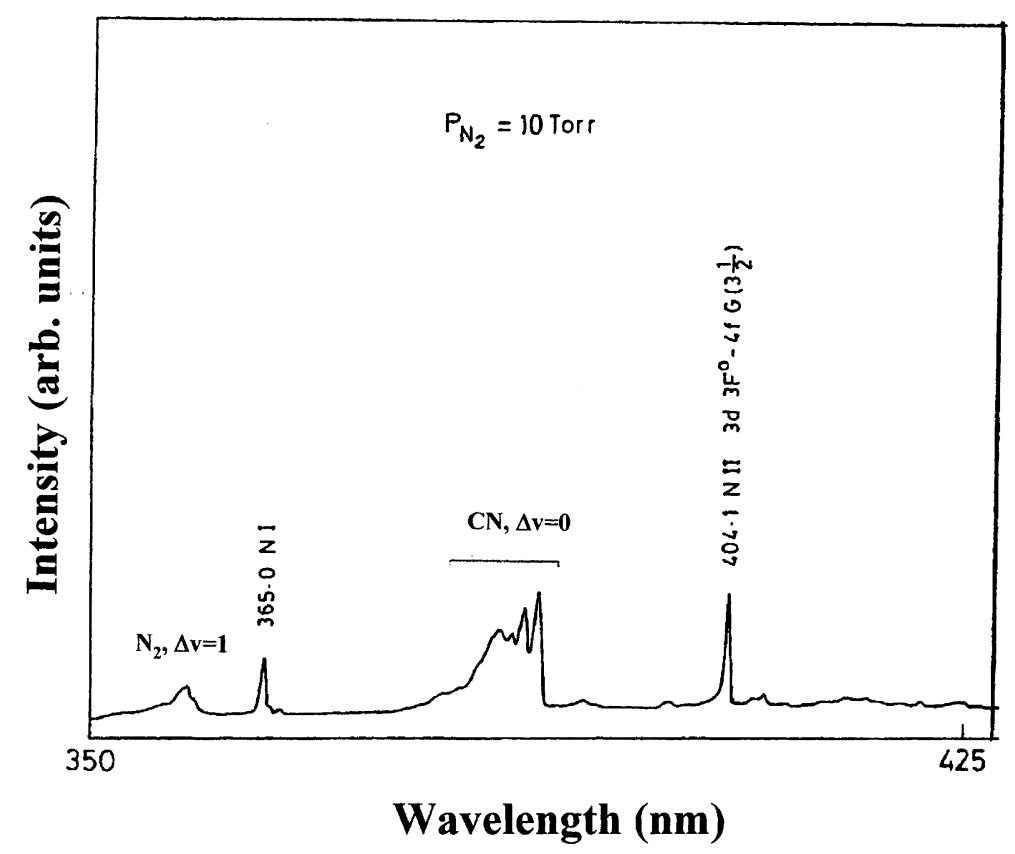

Fig. 7. Emission spectrum of carbon plume at 10 Torr of $\mathrm{N}_{2}$ gas pressure in the spectral range of $350-425 \mathrm{~nm}$ at $10 \mathrm{~mm}$. 


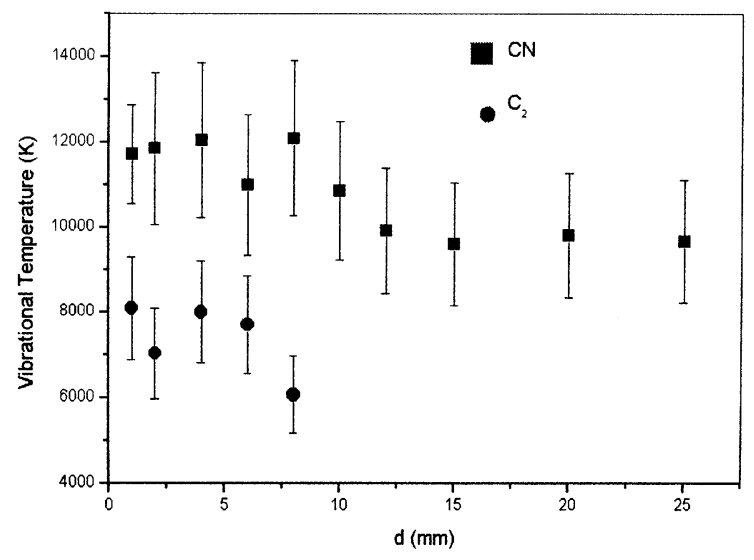

Fig. 8. Variation of vibrational temperature of $\mathrm{C}_{2}$ and $\mathrm{CN}$ with distance from target surface at 1 Torr of nitrogen gas pressure.

relative population in each vibrational level can be found using theoretical Frank-Condon factors and the band intensity. The relative population of the upper vibrational level as derived from the measured intensities was plotted against the vibrational quantum number. The slope of the curve gave the vibrational temperature. Fig. 8 shows the variation of vibrational temperature of $\mathrm{CN}$ and $\mathrm{C}_{2}$ as a function of distance from the target surface at 1 Torr of nitrogen gas pressure. The vibrational temperature of both $\mathrm{CN}$ and $\mathrm{C}_{2}$ species decreases with distance from the target surface. The vibrational temperature of $\mathrm{CN}$ is found to be larger than that of $\mathrm{C}_{2}$. It indicates that the $\mathrm{CN}$ molecules are formed in the energetic collisions between $\mathrm{C}_{2}$ and $\mathrm{N}_{2}$ molecules in the gas phase. The temperature decreases due to collisions of $\mathrm{C}_{2}$ and $\mathrm{CN}$ molecules with the surrounding gas as plasma expands.

The temporal profiles of $\mathrm{CN}$ violet $(0-0)$ band at $388.3 \mathrm{~nm}$ were recorded at different distances from the target surface and nitrogen gas pressures. Fig. 9 shows variation of time delay in the peak emission intensity observed at a distance of $6 \mathrm{~mm}$ from the target surface for various nitrogen gas pressures. The peak emission intensity is found to increase as the pressure is increased to 1 Torr and decreases on further increase in pressure. It further indicates that the formation of $\mathrm{CN}$ radical is optimum at 1 Torr of $\mathrm{N}_{2}$ pressure. The velocity of the plume decreases with increasing collision fre-

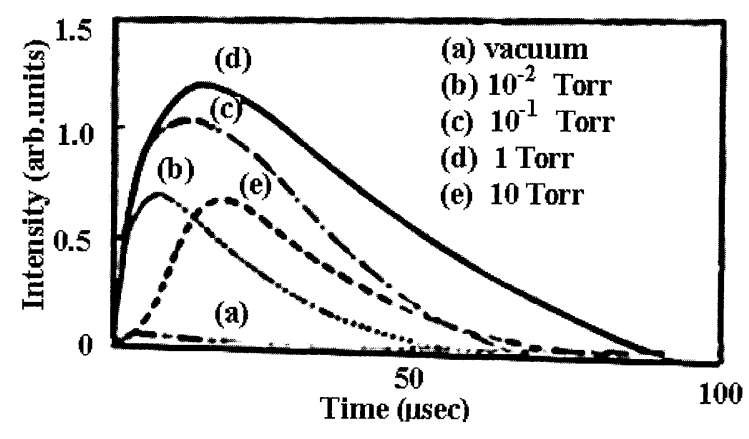

Fig. 9. Temporal profiles of $\mathrm{CN}$ violet band head (0-0) at 388.3 $\mathrm{nm}$ at various nitrogen gas pressures at laser intensity of $1.18 \times 10^{10} \mathrm{~W} \mathrm{~cm}^{-2}$ of $1.064 \mu \mathrm{m}$ radiation.

quency as the ambient gas pressure is increased. Moreover, the confinement of plasma increases the duration of emission.

The above results can be used to understand the formation mechanism of $\mathrm{C}_{2}$ and $\mathrm{CN}$ molecules in a laser-ablated plume in a nitrogen background. $\mathrm{C}_{2}$ molecules in the plasma can originate from different formation mechanisms such as atomic recombination of carbon atoms and ions, fragmentation/dissociation of higher clusters and their ions, electron impact excitation of ground state $\mathrm{C}_{2}$ molecules emitted directly from the target. The observation of atomic/ionic lines in the plasma suggests that $\mathrm{C}_{2}$ is probably formed by atomic recombination of carbon species in presence of background gas by the reaction

$\mathrm{C}+\mathrm{C}+\mathrm{M} \rightarrow \mathrm{C}_{2}+\mathrm{M}$

where $\mathrm{M}$ denotes atoms or molecules of the background gas. In contrast to $\mathrm{C}_{2}$, the maximum in the spatial profile of $\mathrm{CN}$ emission is observed at a distance from the target surface that depends on the background nitrogen pressure. The peaking of intensity of $\mathrm{CN}$ with distance from the target surface indicates that $\mathrm{CN}$ molecule is formed by gas phase reaction between nitrogen gas and carbon plume. The concentration of $\mathrm{CN}$ molecules depends on the density of $\mathrm{C}_{2}$ molecules. Since $\mathrm{C}_{2}$ decreases with distance as shown in Fig. 5 so does $\mathrm{CN}$. The decrease in $\mathrm{C}_{2}$ may be due to decrease in $\mathrm{C}$-atoms in the expanding plume [36]. Thus the density of $\mathrm{CN}$ molecules depends on $\mathrm{C}_{2}$ and the surrounding nitrogen gas. This suggests that $\mathrm{CN}$ is probably formed by a reaction of the form 
$\mathrm{C}_{2}^{*}+\mathrm{N}_{2}=2 \mathrm{CN}^{*}$

The star indicates an excited electronic state of the molecule. Since the bond energies of reactants and products of the reaction are equal, the forward and backward reaction rates are determined by the collision frequency [39]. Therefore, the formation of $\mathrm{CN}$ radical occurs at a point near the target surface where the $\mathrm{C}_{2}$ density is high. The relatively higher bond energy of $\mathrm{CN}$ molecule $(7.8 \mathrm{eV})$ than that of $\mathrm{C}_{2}(6.2 \mathrm{eV})$ increases the chances of its survival in the plasma plume at longer distances, Fig. 5. It also explains the observed peak in emission intensity of $\mathrm{CN}$ in ambient nitrogen gas pressure. The peak position shifts depending on the density of $\mathrm{C}_{2}$ and pressure of the surrounding nitrogen gas. At higher pressures (above 1 Torr), the $\mathrm{CN}$ intensity decrease may be due to dissociation of nitrogen into atomic/ionic nitrogen as seen in difference in spectrum of Fig. 1 and Fig. 7 and the decrease in $\mathrm{C}_{2}$. Our observations clearly imply that the background gas pressure plays a crucial role in defining the laser-ablated plumes.

The peculiar double peak structure observed in Fig. 3 can be attributed to the emission pattern of graphite beyond threshold laser intensity. It is known that graphite exhibits a large difference between the interlayer and intra-layer bond strength and hence at low laser intensities graphite is ablated layer by layer producing large carbon clusters $\left(\mathrm{C}_{n}\right)$ which in turn are dissociated due to collision with electrons emitted from the target. As the laser intensity is increased clusters with lower values are ejected directly from the target. Beyond threshold laser intensity, temperature of the plasma becomes so large in the vicinity of the target that it dissociates $C_{n}$ to neutral and ionized carbon atoms [40]. The absorption due to inverse bremsstrahlung, negligibly small at low laser intensities increases exponentially with increasing laser intensity beyond threshold value [23]. The emission intensity of ionized species increases drastically above the threshold laser intensity $[13,41]$. Once ions and electrons are produced, one can have neutral carbon atoms by three body recombination process of the type $\mathrm{C}^{+}+\mathrm{e}+\mathrm{e}=\mathrm{C}+\mathrm{e}$. However, in the vicinity of the target the temperature of the plume is high and it is the collision pro- cesses, $\mathrm{C}+\mathrm{e}=\mathrm{C}^{+}+\mathrm{e}+\mathrm{e}$ that predominate over the recombination and hence we see excited $\mathrm{C}_{2}$ away from the target. Thus it is expected that beyond a threshold intensity $\mathrm{C}_{2}$ emission will exhibit a twin peak structure corresponding to species generated by dissociation of higher clusters and those generated by recombination. The former peak occurs close to the target and the later farther away from the target. For a given above threshold laser intensity the onset distance (from the target surface) of the double structure depends on the pressure and nature of the ambient gas. As said before since the formation of $\mathrm{CN}$ is dependent on $\mathrm{C}_{2}$, it is expected that $\mathrm{CN}$ will show a twin peak structure appearing in various figures. At high laser intensity Swan band formation has been attributed mainly due to electron-ion and ion-ion recombination [42].

\section{Conclusions}

The laser-ablated plume of carbon in presence of a nitrogen atmosphere is investigated using OES. $\mathrm{C}_{2}$ and $\mathrm{CN}$ bands are found to dominate the emitted spectrum at low laser intensities. It is observed that the laser wavelength, laser energy, and ambient gas pressure greatly influence the plasma emission spectrum. The $\mathrm{C}_{2}$ emission is found to have a narrow spatial profile with a maximum appearing near the target surface whereas $\mathrm{CN}$ emission has a broader spatial profile. Atomic/ ionic lines of nitrogen are prominent at a pressure larger than 1 Torr. The formation of $\mathrm{CN}$ molecule is attributed to the energetic collisions between $\mathrm{C}_{2}$ and nitrogen gas molecules in the gas phase. These studies are useful to elucidate the mechanism involved in the deposition of carbon nitride films by PLD.

\section{Acknowledgements}

Work is partly supported by Council of Scientific and Industrial Research and Department of Science and Technology, New Delhi. RKT acknowledges discussions with Drs. T. Ikegami and Y. Yamagata at the University of Kumamoto, Japan. 


\section{References}

[1] D.G. Lowndes, in: J.C. Miller, R.F. Haglund (Eds.), Laser Ablation and Deposition, Academic Press, San Diego, 1998, p. 551.

[2] D.B. Chrisey, G.H. Hubler (Eds.), Pulsed Laser Deposition of Thin Films, John Willey Sons Inc, New York, 1994.

[3] R.D. Vispute, H. Wu, J. Narayan, Appl. Phys. Lett. 67 (1995) 1549.

[4] M. Okamoto, Y. Mori, T. Sasaki, Jpn. J. Appl. Phys. 38 (1999) 2114.

[5] G.S. Sudhir, H. Fujii, W.S. Wong, C. Kisielowski, N. Newman, C. Dieker, Z.L-. Weber, M.D. Rubin, E.R. Weber, Appl. Surf. Sci. 127-129 (1998) 471.

[6] A. Misra, R.K. Thareja, J. Appl. Phys. 86 (1999) 3438; A. Mishra, R.K. Thareja, Appl. Surf. Sci. 143 (1999) 56.

[7] A.K. Sharma, R.K. Thareja, J. Appl. Phys. 88 (2000) 7334.

[8] Q. Li, X.X. Xi, X.D. Wu, A. Inam, S. Vadlamannath, W.L. McLean, T. Venkatesan, R. Ramesh, D.M. Hwang, J.A. Martinez, L. Nazar, Phys. Rev. Lett. 64 (1990) 3086.

[9] X.Q. Liu, Z.F. Li, W. Lu, S.C. Shen, C.M. Chen, D.Z. Zhu, J. Hu, M.Q. Li, Appl. Phys. Lett. 75 (1999) 2611.

[10] H. Chang, C. Gao, I. Takeuchi, Y. Yoo, J. Wang, P.G. Schultz, X.-D. Xiang, R.P. Sharma, M. Downes, T. Venkatesan, Appl. Phys. Lett. 72 (1998) 2185.

[11] R.K. Dwivedi, S.P. Singh, R.K. Thareja, Int. J. Mod. Phys. B 12 (1998) 2619;

R.K. Dwivedi, S.P. Singh, R.K. Thareja, Phys. Rev. B 51 (1995) 7160

[12] M.Y. Chen, P.T. Murray, J. Vac. Sci. Technol. A 16 (1998) 2093.

[13] Abhilasha, P.S.R. Prasad, R.K. Thareja, Phys. Rev. E 48 (1993) 2929.

[14] J. Muramota, I. Sakamota, Y. Nakata, T. Okada, M. Maeda, Appl. Phys. Lett. 75 (1999) 751.

[15] E.A. Rohlfing, D.M. Cox, A. Kaldor, J. Chem Phys. 81 (1984) 3322

[16] P.S.R. Prasad, Abhilasha, R.K. Thareja, Phys. Stat. Sol. (a) 139 (1993) K1.

[17] P.R. Willmott, R. Timm, J.R. Huber, J. Appl. Phys. 82 (1997) 2028.

[18] J. Neamtu, I.N. Mihailescu, C. Ristoscu, J. Hermann, J. Appl. Phys. 86 (1999) 6096
[19] Y. Matsuo, T. Nakajima, T. Kobayashi, M. Takami, Appl. Phys. Lett. 71 (1997) 996.

[20] V. Berardi, S. Amoruso, N. Spinelli, M. Armenante, R. Vellotta, F. Fuso, M. Allegrini, E. Arimondo, J. Appl. Phys. 76 (1994) 8077.

[21] R. Timm, P.R. Willmott, J.R. Huber, J. Appl. Phys. 80 (1996) 1794.

[22] P.R. Willmott, J.R. Huber, Rev. Mod. Phys. 72 (2000) 315.

[23] T.P. Hughes, Plasma and Laser Light, Wiley, New York, 1975.

[24] G. Bekfi, Principles of Laser Plasmas, Wiley, New York, 1976.

[25] J. Dawson, P.K. Kaw, B. Green, Phys. Fluids 12 (1969) 875.

[26] R.K. Singh, J. Narayan, Phys. Rev. B 41 (1990) 8843.

[27] A. Vertes, M. De Wolf, P. Juhasz, R. Gijbels, Anal. Chem. 61 (1989) 1029.

[28] J.C.S. Kools, J. Appl. Phys 74 (1993) 6401.

[29] S. Franklin, R.K. Thareja, Appl. Surf. Sci. 177 (2001) 15.

[30] P.E. Dyer, A. Issa, P.H. Key, Appl. Phys. Lett. 57 (1990) 186.

[31] D.B. Geohegan, A.A. Puretzky, Appl. Phys. Lett. 67 (1995) 197.

[32] P.E. Dyer, J. Sidhu, J. Appl. Phys. 64 (1986) 4657.

[33] L. St-Onge, R. Sing, S. Bechard, M. Sabsabi, Appl. Phys. A 69 (1999) 5913.

[34] R.W.B. Pearse, A.G. Gaydon, The Identification of Molecular Spectra, 4th ed., Chapman and Hall, London, 1976.

[35] A.R. Striganov, N.S. Sventitskii, Tables of Spectral Lines of Neutral and Ionized Atoms, Plenum, New York, 1968.

[36] S.M. Park, K.H. Lee, Appl. Surf. Sci. 178 (2001) 37.

[37] P.T. Rumsby, J.W.M. Paul, Plasma Phys. 16 (1974) 247.

[38] R.K. Thareja, Abhilasha, J. Chem. Phys. 100 (1994) 4019.

[39] C. Vivien, J. Hermann, A. Perrone, C. Boulmer-Leborgne, A. Luches, J. Phys. D: Appl. Phys. 31 (1998) 1263.

[40] Y. Iida, E.S. Yeung, Appl. Spect. 48 (1994) 945.

[41] S.S. Harilal, R.C. Issac, C.V. Bindhu, V.P.N. Nampoori, C.P.G. Vallabhan, J. Appl. Phys. 80 (1996) 3561.

[42] D.L. Pappas, K.L. Saenger, J.J. Cuomo, R.W. Dreyfus, J. Appl. Phys. 72 (1992) 3966. 